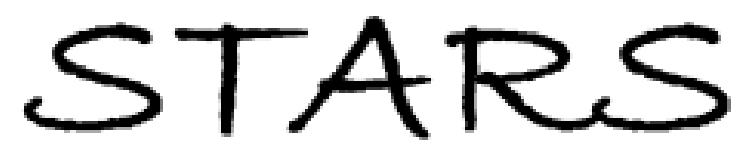

University of Central Florida

STARS

$1-1-2006$

\title{
Spatially tunable laser emission in dye-doped photonic liquid crystals
}

Yuhua Huang

University of Central Florida

Ying Zhou

University of Central Florida

Shin-Tson Wu

University of Central Florida

Find similar works at: https://stars.library.ucf.edu/facultybib2000

University of Central Florida Libraries http://library.ucf.edu

This Article is brought to you for free and open access by the Faculty Bibliography at STARS. It has been accepted for inclusion in Faculty Bibliography 2000 s by an authorized administrator of STARS. For more information, please contact STARS@ucf.edu.

\section{Recommended Citation}

Huang, Yuhua; Zhou, Ying; and Wu, Shin-Tson, "Spatially tunable laser emission in dye-doped photonic liquid crystals" (2006). Faculty Bibliography 2000s. 6241.

https://stars.library.ucf.edu/facultybib2000/6241

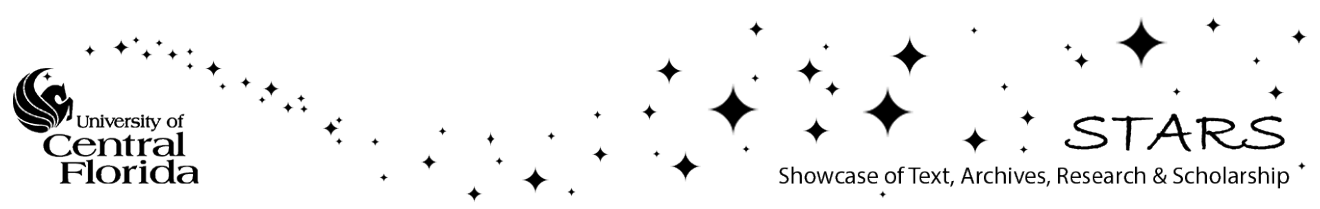




\title{
Spatially tunable laser emission in dye-doped photonic liquid crystals
}

\author{
Yuhua Huang, Ying Zhou, and Shin-Tson Wua \\ College of Optics and Photonics, University of Central Florida, Orlando, Florida 32816
}

(Received 15 September 2005; accepted 5 December 2005; published online 3 January 2006)

\begin{abstract}
A spatially tunable laser emission of the dye-doped cholesteric liquid crystal (CLC) cell using a one-dimensional temperature gradient is demonstrated. The photoexcitation of dye-doped CLC device using a frequency-doubled pulsed $\mathrm{Nd}$ : yttrium-aluminium-garnet laser gives rise to laser emission in the yellow-red spectral range. The lasing wavelength is widely tunable from 577 to 670 $\mathrm{nm}$ by shifting the position of the dye-doped CLC cell with respect to the pumping beam. The lowest excitation energy and maximum lasing efficiency occur at $\lambda \sim 605 \mathrm{~nm}$ which corresponds to the peak fluorescence emission of the dye. (C) 2006 American Institute of Physics.
\end{abstract}

[DOI: $10.1063 / 1.2161167]$

Liquid crystals (LCs) doped with chiral molecules exhibit a self-organized periodic helical structure. Since LC is a highly birefringent medium, the periodic helical structure gives a periodic modulation of the refractive index. Consequently, a one-dimensional photonic band gap (PBG) is established with the central wavelength at $\lambda=n p$, where $p$ is the helical pitch and $n$ the average refractive index. Cholesteric liquid crystals (CLCs) have many unique properties including supramolecular helicoidal periodic structure (the period can be set in a wide range from $100 \mathrm{~nm}$ up to infinity), $100 \%$ selective reflection of a circularly polarized light and the ability of shifting the selective reflection wavelength by external factors including electric, magnetic, acoustic fields, temperature, and light irradiation. ${ }^{1-5}$ Because of these properties, CLCs have attracted great interest for mirrorless laser applications. Among these properties, the most interesting feature is that the helical pitch can be modified by means of an external field, which provides the possibility of assembling tunable mirrorless lasers. The tunability of lasing in the CLC systems has been achieved using various methods. For instance, a phototunable laser has been demonstrated using ultraviolet (UV) radiation based on the change of the chiral's twist power under the UV illumination. ${ }^{2,6,7}$ A tunable lasing emission was observed by changing the temperature of the CLC cell. ${ }^{8}$ A spatially turnable laser was obtained by filling several CLC mixtures with different chiral concentration into the same cell one by one. 9,10

In this letter, we have demonstrated a spatially tunable lasing emission by generating one-dimensional (1D) temperature gradient in a dye-doped CLC cell. Different from the abovementioned spatially tunable methods, our CLC cell contains only one mixture. The spatial tunability originates from the temperature dependent CLC PBG. The laser emission wavelength can be tuned from 577 to $670 \mathrm{~nm}$ by changing the spatial position (within $\sim 3 \mathrm{~mm}$ ) of the dye-doped CLC cell with respect to the pump beam [a frequencydoubled pulsed Nd: yttrium-aluminium-garnet (YAG) laser].

The CLC host was prepared by mixing $66 \mathrm{wt} \%$ BL006 (Merck LC mixture) with 34 wt\% of a left-handed (LH) chiral dopant ZLI-811 (also from Merck). Afterwards, 1 wt $\%$ of laser dye 4-(dicyanomethylene)-2-methyl-6-(4-

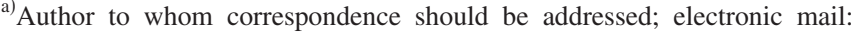
swu@mail.ucf.edu
}

dimethylaminostryl)-4H-pyran (DCM, Exciton) was added to the mixture. The mixture was stirred in isotropic phase for $\sim 4 \mathrm{~h}$ to make the constituents uniformly mixed and then capillary filled in the isotropic phase into a 10 - $\mu \mathrm{m}$-thick LC cell. Inside the cell, both the glass substrates were coated with a thin polyimide alignment layer. The anti-parallel rubbing-induced pretilt angle is $\sim 3^{\circ}$. After the temperature was gradually cooled down to room temperature, a CLC sample with LH helix was formed.

First, we characterized the temperature dependent photonic band gap spectrum of the dye-doped CLC sample by changing the temperature from 23 to $40{ }^{\circ} \mathrm{C}$. The temperature of the dye-doped CLC cell was monitored by a temperature controller. Figure 1 shows the shift in the PBGs of the dyedoped CLC cells with the increased temperature. The average wavelength $\left(\lambda_{\text {ave }}\right)$ is defined as $\lambda_{\text {ave }}=\left(\lambda_{\text {long }}+\lambda_{\text {short }}\right) / 2$, where $\lambda_{\text {long }}$ and $\lambda_{\text {short }}$ stand for the wavelength at the long and short reflection band edges, respectively. As the temperature of the CLC cell increases, the selective reflection band of CLC shifts toward a shorter wavelength. The reason is that at room temperature, the maximum solubility of ZLI-811 chiral agent in BL-006 is $\sim 25 \mathrm{wt} \%$. However, our sample contains $34 \mathrm{wt} \%$ ZLI-811 which is beyond the maximum solubility. Therefore, a portion of the chiral ZLI-811 cannot be dissolved in the LC host and is separated out. As the temperature increases, the solubility of the chiral agent increases which, in turn, reduces the helical pitch length. As a result, the PBG shifts towards a shorter wavelength as the temperature increases. At $T>40{ }^{\circ} \mathrm{C}$, nearly all the chiral dopants have been dissolved and, therefore, no more blueshift occurs as the temperature is further increased.

Based on the temperature dependent optical properties of the CLC sample, we can easily obtain a spatially tunable CLC PBG by generating a 1D temperature gradient across the CLC cell. To achieve this goal, we simply placed one side of the CLC cell on a heating stage and left the other side in the air. By raising the temperature of the heating stage over room temperature, the $1 \mathrm{D}$ temperature gradient was formed. Figure 1(b) shows the image of a CLC cell with gradient temperature at $T \sim 50{ }^{\circ} \mathrm{C}$. The reflected colors spread from blue to red as the position gets farther away from the heat source.

An important application of the tunable PBG in the CLC system is for tuning the laser oscillation. To test the laser emission properties, a second-harmonic $Q$-switched Nd:YAG 

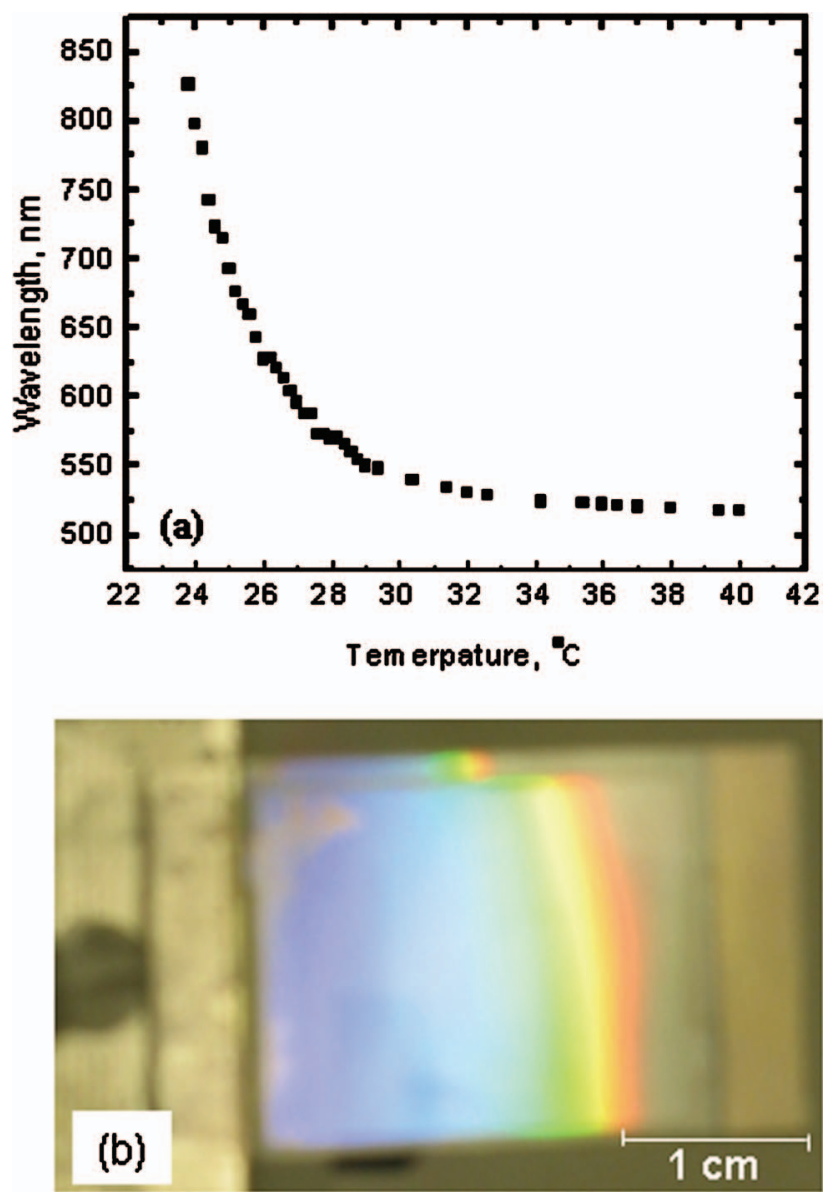

FIG. 1. (Color) The measured average reflection wavelength of the dyedoped CLC cell as a function of the temperature (a) and image of the gradient CLC reflection band (b).

pulsed laser (from Continuum) at $\lambda=532 \mathrm{~nm}$ with vertical linear polarization was used to excite the dye-doped CLC sample, as Fig. 2 depicts. The pulse width and repetition rate are $6 \mathrm{~ns}$ and $1 \mathrm{~Hz}$, respectively. The reason that we chose 1 $\mathrm{Hz}$ repetition rate is to avoid sample heating and degradation. A beam splitter was used to divide the incoming laser into two beams: one was sent to a laser energy meter (Ophir) for monitoring the pumping pulse energy and the other used as the excitation beam. A linear polarizer and a quarter-wave plate were used to convert the linear polarization into righthanded circular polarization to avoid the reflection by the CLC PBG. A lens with $15 \mathrm{~cm}$ focal length focused the incident beam to a small spot of $\sim 160 \mu \mathrm{m}$ diameter at the sample. One side of the sample was placed on a heating stage $\left(T=50{ }^{\circ} \mathrm{C}\right)$ and the other was left in the air. The heating

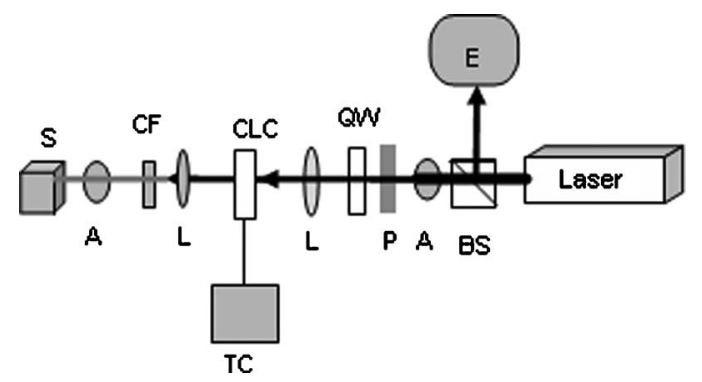

FIG. 2. The schematic of experimental setup. A: attenuator; BS: beam splitter; $\mathrm{CF}$ : color filter; E: energy meter; L: lens; P: polarizer; QW: quarter wave plate; S: spectrometer; TC: temperature controller.
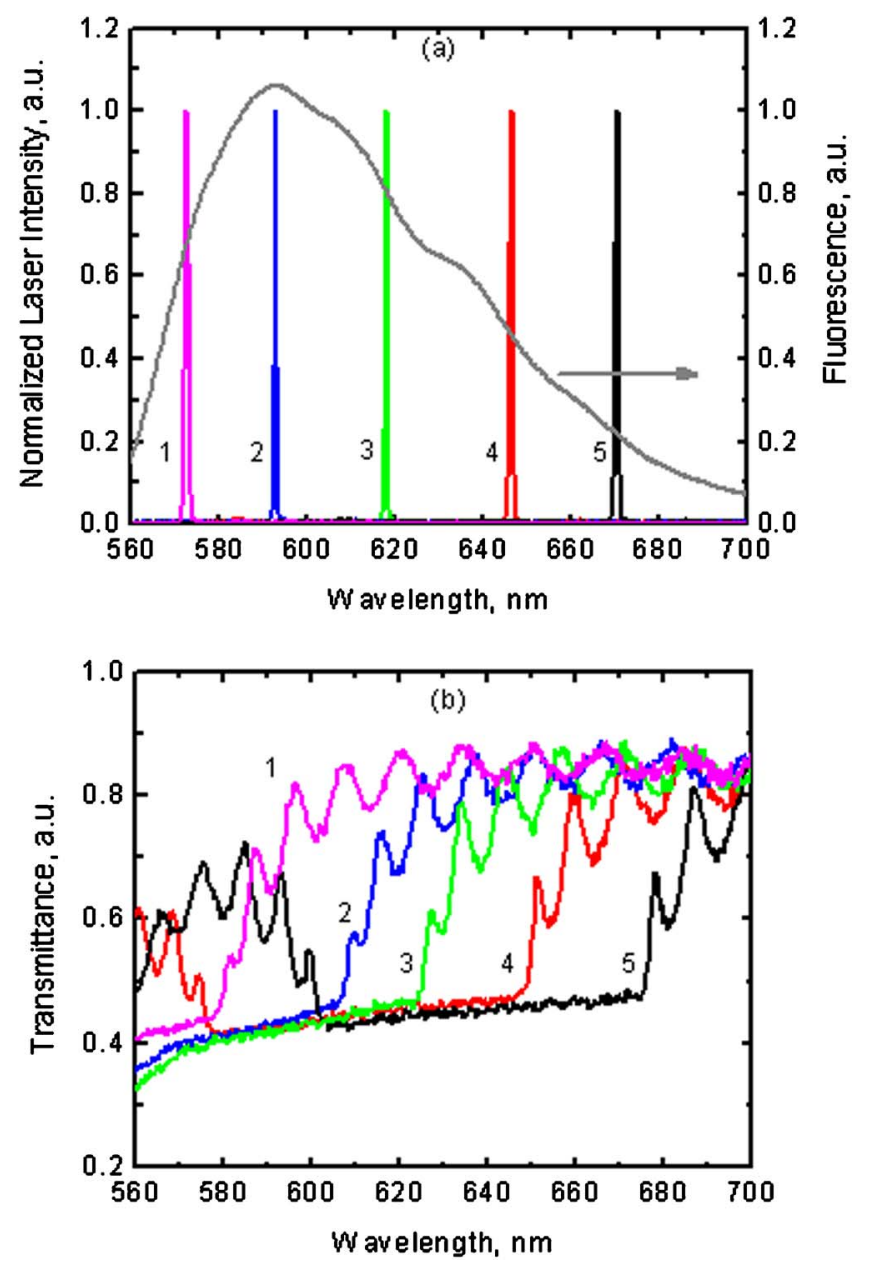

FIG. 3. (Color online) (a) Normalized laser emission and (b) the corresponding transmission spectra of the dye-doped CLC cells with various PBGs at different sample positions. Positions $1-5$ correspond to $T \sim 30.6$, $28.8,27.6,26.8$, and $26.0^{\circ} \mathrm{C}$, respectively. The gray line represents the dye's fluorescence spectrum. The laser emission occurs at band edge.

stage was mounted on a translational stage. The output laser emission in the forward direction of the sample was collected by a lens to a fiber-optics based universal serial bus (USB) spectrometer (0.04 $\mathrm{nm}$ resolution; USB HR2000, Ocean Optics).

In Figs. 3(a) and 3(b), we plot the normalized laser emission and the corresponding PBGs spectra, respectively, of the dye-doped CLC cells at different positions, i.e., different temperatures. For instance, positions 1-5 correspond to $T \sim 30.6,28.8,27.6,26.8$, and $26^{\circ} \mathrm{C}$, respectively. In principle, the emitted laser intensity should be related to the gain of the media, which is proportional to the dye's fluorescence spectrum. Here, our purpose is to show the trend of the lasing wavelength with respect to the temperature gradient rather than the lasing efficiency. To avoid the crowdedness of the figure, we just select five lasing wavelengths in Fig. 3(a) and their corresponding CLC PBGs in Fig. 3(b). The laser emission peak shifts noticeably according to the temperature gradient of the CLC cell. The lasing wavelength shifts from $\lambda=577 \mathrm{~nm}$ at the side close to the heating stage to $670 \mathrm{~nm}$ at the farther side. Under such a circumstance, the tuning of laser wavelength can be achieved through the temperature gradient of the CLC PBG.

Although the CLC PBG can be widely tuned from $\lambda \sim 500$ to $\sim 820 \mathrm{~nm}$, the laser emission was only observed 
between 577 and $670 \mathrm{~nm}$. This behavior stems from the competition between the optical loss and gain in the CLC system. Generally, the laser emission can be generated through the feedback amplification only when the optical gain inside the medium is larger than the loss. For the CLC cell, since the internal distributed feedback of the CLC PBG provides the amplification of the optical gain traveling in the CLC cell, the laser emission can be obtained when the amplification of the optical gain traveling in the CLC cell is sufficient to overcome the losses in the medium. Typically, the optical gain spectrum has the same spectral appearance as the fluorescence spectrum of the dye. When the CLC PBG is falling within the fluorescence spectrum of the doped dye, the optical emission can be effectively obtained by the internal distributed feedback of the CLC PBG. Therefore, we can obtain laser emission in this range. However, when the CLC PBG is scarcely overlapped with the fluorescence spectrum of the doped dye, the amplification of the optical emission provided by the internal distributed feedback of the CLC PBG cannot yet overcome the optical losses. Thus, no lasing can be observed. It was also observed that the laser emission always appears at the long edge of the CLC PBG because the long edge of the CLC PBG needs lower energy than the short edge to get laser emission.

In addition, we also investigated the dependence of the lasing emission intensity at different wavelengths on the excitation energy. Figure 4 shows the results we obtained from the dye-doped CLC cell with a PBG at different spectral position by adjusting the position of the dye-doped CLC with respect to the pump beam. For any lasing wavelengths, the emission intensity is significantly enhanced as the excitation energy exceeds the threshold. However, the threshold excitation energy and lasing efficiency strongly depend on the lasing wavelength, as Fig. 4 shows. When the lasing wavelength is at $\lambda=605 \mathrm{~nm}$, the lasing efficiency reaches the maximum $(\sim 0.5 \%)$ and threshold excitation energy is the lowest $(\sim 4.7 \mu \mathrm{J} /$ pulse $)$. If the lasing wavelength is away from $605 \mathrm{~nm}$, higher threshold excitation energy is required and the lasing efficiency is decreased. We know that laser emission can be obtained only when the optical gain overcomes the losses in the medium and larger optical gain makes the generation of the laser emission easier because the optical emission is more effectively obtained by the feedback effect. As stated above, the optical gain spectrum relies on the fluorescence spectrum of the dye. Since the DCM dye exhibits its maximum fluorescence at $\lambda \sim 605 \mathrm{~nm}$, the lowest threshold excitation energy of $\sim 4.7 \mu \mathrm{J} /$ pulse and the highest lasing efficiency occur for the dye-doped CLC sample when the long edge of the PBG is at $605 \mathrm{~nm}$. When the long edge of the CLC PBG overlaps with the tail of the fluorescence band, higher threshold energy is required for exciting more optical emission to overcome the loss and finally generating the laser feedback effect. Consequently, the lasing efficiency is decreased.

In conclusion, we have demonstrated a spatially tunable laser emission by generating a 1D temperature gradient on a thermally sensitive dye-doped CLC cell. The lasing wavelength is tunable from 577 to $670 \mathrm{~nm}$ by changing the spatial
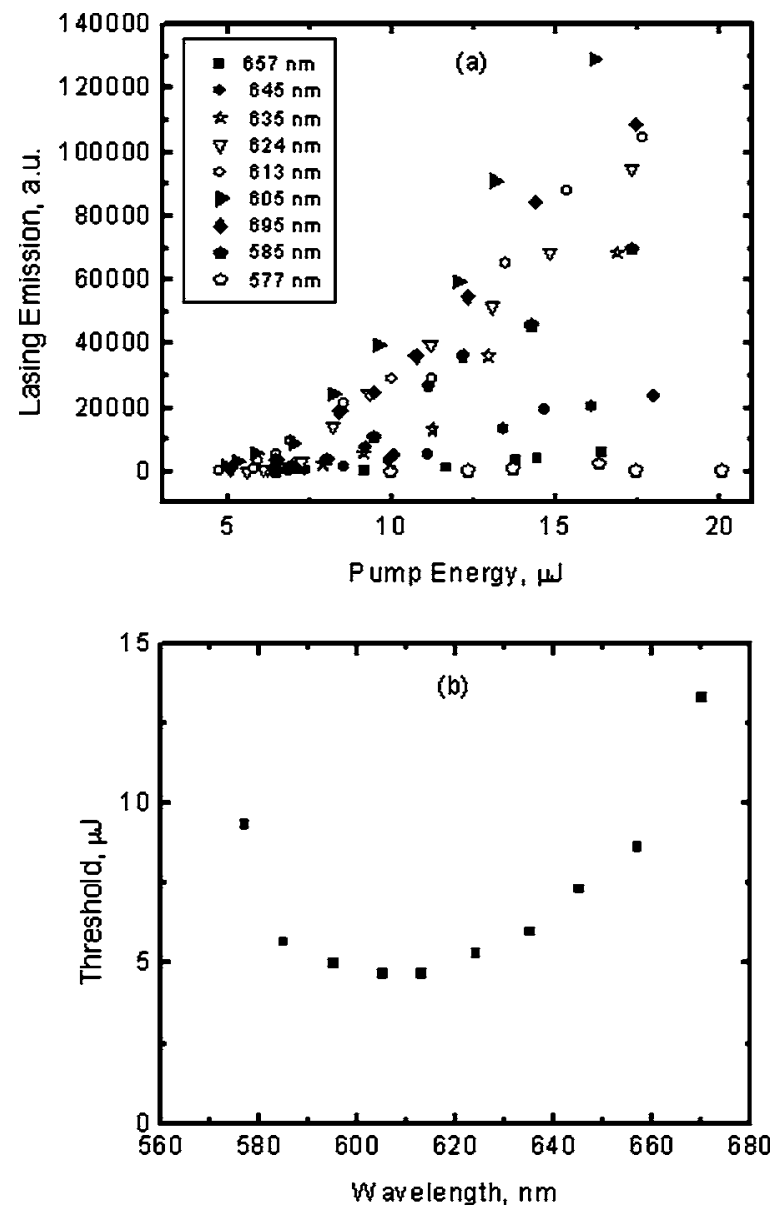

FIG. 4. The laser emission intensity from the dye-doped CLC cell as a function of: (a) the excitation energy of the pumping beam at $532 \mathrm{~nm}$ and (b) the threshold excitation energy as a function of the lasing wavelength.

position of the dye-doped CLC cell. The lowest excitation energy and maximum lasing efficiency are obtained at $\lambda \sim 605 \mathrm{~nm}$ since the DCM dye has the maximum fluorescence emission at around $605 \mathrm{~nm}$.

${ }^{1}$ S. Furumi, S. Yokoyama, A. Otomo, and S. Mashiko, Appl. Phys. Lett. 82, 16 (2003).

${ }^{2}$ A. Chanishvili, G. Chilaya, G. Petriashvili, R. Barberi, R. Bartolino, G. Cipparrone, A. Mazzulla, and L. Oriol, Appl. Phys. Lett. 83, 5353 (2003).

${ }^{3}$ S. M. Morris, A. D. Ford, M. N. Pivnenko, and H. J. Coles, J. Appl. Phys. 97, 023103 (2005).

${ }^{4}$ Y. D. Ma and B. G. Wu, US patent No. 5,949,513 (1999).

${ }^{5}$ L. C. Chien, U. Muller, M. F. Nabor, and J. W. Doane, SID Tech. Digest 26, 169 (1995).

${ }^{6}$ S. Furumi, S. Yokoyama, A. Otomo, and S. Mashiko, Appl. Phys. Lett. 84, 2491 (2004).

${ }^{7}$ T. H. Lin, Y. J. Chen, C. H. Wu, A. Y. G. Fuh, J. H. Liu, and P. C. Yang, Appl. Phys. Lett. 86, 161120 (2005).

${ }^{8}$ K. Funamoto, M. Ozaki, and K. Yoshino, Jpn. J. Appl. Phys., Part 2 42, L1523 (2003).

${ }^{9}$ A. Chanishvili, G. Chilaya, G. Petriashvili, R. Barberi, R. Bartolino, G. Cipparrone, A. Mazzulla, and L. Oriol, Adv. Mater. (Weinheim, Ger.) 16, 791 (2004).

${ }^{10}$ A. Chanishvili, G. Chilaya, G. Petriashvili, R. Barberi, R. Bartolino, G. Cipparrone, A. Mazzulla, R. Gimenez, L. Oriol, and M. Pinol, Appl. Phys. Lett. 86, 051107 (2005). 